\title{
Case of hibernoma in the right supraclavicular fossa
}

\author{
Marilyn Neufeld BSc ${ }^{1}$, Shaun Medlicott MD², Duncan Nickerson BSc MD FRCSC ${ }^{3}$
}

M Neufeld, S Medlicott, D Nickerson. Case of hibernoma in the right supraclavicular fossa. Can J Plast Surg 2005;13(1):33-35.

A patient with a supraclavicular mass originally diagnosed as a lipoma who was referred to a plastic surgeon for definitive treatment is presented. Gross intraoperative findings were inconsistent with lipoma, and subsequent pathological evaluation revealed the mass to be a hibernoma (a benign, brown adipose tumour). The incidence, etiology, presentation, pathology and imaging characteristics of this somewhat rare soft tissue tumour are reviewed.

\section{Un cas d'hibernome dans la fosse supraclaviculaire droite}

\begin{abstract}
Est exposé le cas d'un patient présentant une masse supraclaviculaire d'abord diagnostiquée comme un lipome et aiguillé vers un plasticien en vue d'un traitement définitif. Les observations macroscopiques peropératoires ne corroboraient pas la présence d'un lipome, et l'évaluation pathologique subséquente a révélé que la masse était un hibernome (tumeur adipeuse brunâtre bénigne). L'incidence, l'étiologie, la présentation, la pathologie et les caractéristiques d'imagerie de cette tumeur plutôt rare des tissus mous sont analysées.
\end{abstract}

Key Words: Fatty tumour; Hibernoma; Lipoma; Pleomorphic

A

43-year-old woman presented with a right neck mass that had been noticeably expanding for six months. She had undergone an incisional biopsy by a primary care physician. The results were consistent with lipoma, and she was therefore referred to a plastic surgeon for definitive treatment.

Physical examination revealed an approximately $8 \mathrm{~cm}$ diameter, nonpainful, smooth, mobile, unilobular mass in the right supraclavicular fossa. Overlying it was a well-healed scar from a previous incisional biopsy. The clinical impression was thought to be compatible with the incisional biopsy finding of lipoma. Complete excision was offered. Due to the location and size of the mass, excision under general anesthesia was proposed, and accepted by the patient.

Under general anesthesia, a curvilinear incision was developed directly over the mass. No mass was encountered in the subcutaneous plane. The mass was palpable deep to the platysma muscle. The platysma was divided in line with its fibres and the mass was encountered.

Several characteristics were immediately noted that set this mass apart from a typical lipoma. Specifically, it was dark brown in colour, in contrast to the bright yellow of a typical lipoma. Additionally, whereas a typical lipoma separates readily from adjacent tissues with spreading or blunt dissection, this mass was less well-defined and more adherent to adjacent tissues. It was also extremely vascular, with numerous feeding vessels requiring ligation, unlike a typical lipoma. Eventually, the mass was freed from most of the adjacent tissues, and followed toward a tail that extended deep to the anterior, superior edge of the right trapezius muscle. It was thought that the existing exposure did not permit safe exposure deep to the trapezius and the deepest portion of the tail (less than $1 \mathrm{~cm}$ in cross-sectional area) was simply transected, yielding a glistening brown mass measuring $9 \mathrm{~cm} \times 6 \mathrm{~cm} \times 7 \mathrm{~cm}$.

The wound was closed over a suction drain and the patient was discharged later that day. The suction drain was removed after approximately $36 \mathrm{~h}$. The wound healed uneventfully, and three months postoperatively the patient was symptom free without clinical evidence of residual or recurrent mass.

Gross pathological evaluation revealed multilobulated dark yellow fat with no areas of hemorrhage, necrosis or induration. Histology later confirmed the diagnosis of hibernoma, with multivacuolated and univacuolated cells and no cellular atypia or mitoses (Figures 1 and 2).

\section{DISCUSSION}

Hibernoma is a rare tumour composed of brown adipose tissue, and represents less than $2 \%$ of benign lipomatous tumours (1). This tumour was first described by Merkel in 1906 as a "pseudolipoma" and it was later named "hibernoma" in 1914 (2).

Approximately 150 cases had been reported worldwide until Furlong et al (3) reported 170 cases from the Armed Forces Institute of Pathology in 2001. This series revealed a slightly higher prevalence of the tumour in males (58\%) and a mean patient age of 38 years. The tumour was most commonly located at the thigh (29\%), shoulder (12\%) and back (10\%), followed by the neck (9\%), chest (6\%), arm (6\%) and abdominal cavity/retroperitoneum (6\%). The size of the tumours varied from $1 \mathrm{~cm}$ to $24 \mathrm{~cm}$ and they were completely excised without recurrence at a mean follow-up of 7.7 years (range of six months to 28 years).

To our knowledge, only three cases in the supraclavicular fossa have been documented (4).

${ }^{1}$ Faculty of Medicine; ${ }^{2}$ Department of Pathology; ${ }^{3}$ Department of Surgery, Division of Plastic Surgery, University of Calgary and Calgary Regional Health Authority, Calgary, Alberta

Correspondance and reprints: Dr Duncan Nickerson, Suite 140, 1402 8th Avenue Northwest, Calgary, Alberta T2N 1 B9.

Telephone 403-282-6800, fax 403-282-6890, e-mail duncan.Nickerson@ehealthab.ca 


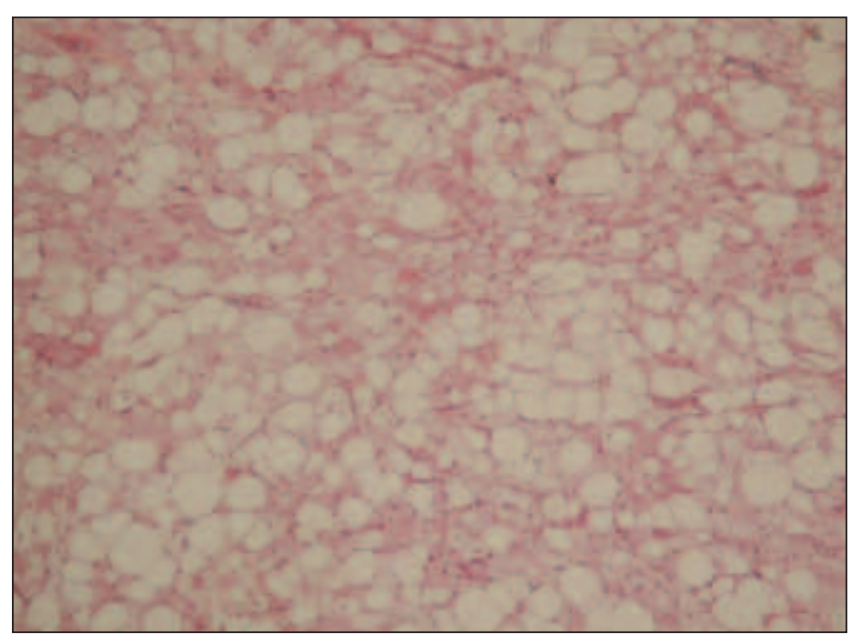

Figure 1) Microphotograph showing hibernoma architecture as a solid sheet of bland adipocytes, the majority having the characteristic eosinophilic cytoplasm (hematoxylin and eosin stain, original magnification $\times 4$ )

Brown adipose tissue promotes shivering thermogenesis in the fetus and newborn humans through the action of the mitochondrial uncoupling protein, which uncouples the respiratory chain from ATP synthesis, allowing heat production (5). This unique form of energy production was associated with a $16 \mathrm{~kg}$ weight loss in a 47-year-old man with a flank hibernoma (6).

Brown adipose tissue persists as a vestigial organ at the neck, axilla, mediastinum, periaortic and perirenal zones in humans, but is also present at the interscapular, peripancreatic and suprailiac regions, as well as surrounding the sympathetic ganglia in fetuses (7). Furlong et al's series (3) has shown that hibernomas are most prevalent in the lower extremities. This would tend to dispute the notion that hibernomas develop as vestigial brown fat, and rather would suggest a neoplastic pathogenesis because vestigial brown fat is not typically found in the lower extremities.

Grossly, hibernomas are brown-red, soft and encapsulated, with a yellow centre due to the vacuolated cells within. Histological and cytological analyses reveal variable amounts of mature, white adipocytes as well as three characteristic brown fat cell types including a granular eosinophilic cell with or without lipid vacuoles; large univacuolated adipocytes with eccentric nuclei; and multivacuolated cells with scant granular eosinophilic cytoplasm and small nuclei (7). There are four morphological variants of the multivacuolated cells including typical, myxoid, spindle cell and lipomalike, which may stain positive for $\mathrm{p} 53$ and S-100 proteins $(3,7,8)$. CD -34 positivity may also be seen in the spindle variants (3). Cells are arranged in lobules with septations and multiple capillaries giving the tumour its vascular appearance $(7,8)$.

Complex karyotypical changes involving chromosome 11q13 q21 have recently been identified in hibernomas $(1,9)$. $\mathrm{Up}$ to $4 \mathrm{Mbp}$ of heterozygous and homozygous deletions of chromosome 11 have been detected, involving PPP1A and MEN1, respectively. Although the tumours of multiple endocrine neoplasia type 1 (MEN1) and hibernomas share the MEN1 deletion, their pathogenesis is likely different. MEN1 tumours have malignant potential whereas hibernomas do not (3).

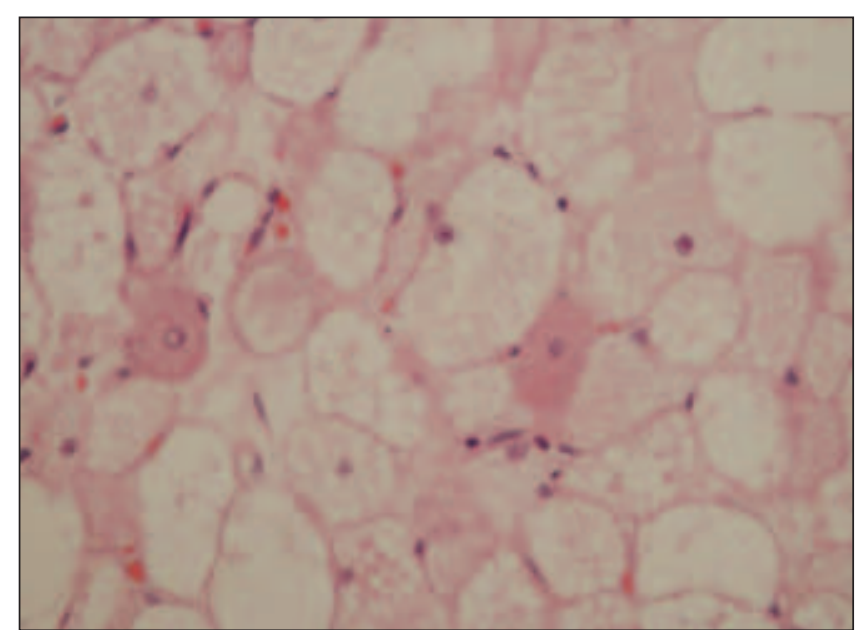

Figure 2) Cytology of the neoplasm is an admixture of translucent mature adipocytes and brown fat cells with granular eosinophilic cytoplasm. Many of the latter cells are multivacuolated with compartmentalized fat (hematoxylin and eosin stain, original magnification $\times 10$ )

There may also be a second tumour suppressor, PPP1A, downstream that is deleted in hibernoma. Mutations near the GARP gene have been identified as well as translocations with chromosomes 5, 16 and 22, involving PYGM, MEN1, CCND1, FGF3, ARIX and GARP $(1,9)$.

Ultrasonography of these tumours typically shows a homogenous, hypoechoic and hypervascular mass $(2,10)$. Angiography is a useful tool due to the hypervascularity of the lesion and may show arteriovenous shunting and neovascularity $(10,11)$. Computed tomography shows a solid, septated, vascular mass with fatty density and variable contrast enhancement $(2,12)$. Magnetic resonance imaging is highly specific for diagnosing lipomatous tumours, including hibernomas. T1 weighted images show a hypodense mass (between skeletal muscle and subcutaneous fat), and T2 images show a heterogenous signal intensity approaching that of subcutaneous fat $(2,10,12)$. Due to the difficulty in differentiating hibernomas from welldifferentiated liposarcomas, complete excision of the mass is still recommended for definitive diagnosis and treatment (10).

At surgery, hibernomas may displace normal tissue but are unlikely to invade adjacent structures. Intermuscular masses may also be encountered (12). Partial excision may result in recurrence leading to the recommendation for complete excision (7); however, due to the benign nature of the mass, partial excision may be acceptable if there is a significant risk of neurovascular compromise at the time of surgery (11). There is no role for adjuvant therapy in treatment.

In the present case, a previous incisional biopsy yielded the diagnosis of lipoma. We strongly believe that this is likely a case of sampling error. Our patient's tumour was deep to the platysma and normal subcutaneous fat was most probably biopsied. Albeit unusual, a progressively expansile mass of the neck warrants consideration of hibernoma.

\section{REFERENCES}

1. Sandberg AA. Updates on the cytogenetics and molecular genetics of bone and soft tissue tumors: Liposarcoma. Cancer Genet Cytogenet 2004;150:93-115.

2. Ong SY, Maziak DE, Shamji FM, Matzinger FR, Perkins DG. Intrathoracic hibernoma. Can J Surg 2002;45:145-6. 
3. Furlong MA, Fanburg-Smith JC, Miettinen M. The morphologic spectrum of hibernoma: A clinopathologic study of 170 cases. Am J Surg Path 2001;25:809-14.

4. Sugiura K, Sugiura M, Hayakawa R, Shamoto M. Hibernoma in the left scapula. Skinmed 2003;2:193-4.

5. Carinci F, Carls FP, Pelucchi S, Grandi E, Hassanipour A, Pastore A. Hibernoma of the neck. J Craniofac Surg 2001;12:284-6.

6. Essadel A, Bensaid Alaoui S, Mssrouri R, et al. Hibernoma: A rare case of massive weight loss. Ann Chir 2002;127:215-7.

7. Lele SM, Chundru S, Chaljub G, Adegboyega P, Haque AK. Hibernoma. A report of 2 unusual cases with a review of the literature. Arch Pathol Lab Med 2002;126:975-8.
8. Saqi A, Yu GH, Marshall MB. Fine-needle aspiration of hibernoma. Diagn Cytopathol 2003;29:44-5.

9. Maire G, Forus A, Foa C, et al. 11q13 alterations in two cases of hibernoma: Large heterozygous deletions and rearrangement breakpoints near GARP in 11q13.5. Genes Chromosomes Cancer 2003;37:389-95.

10. Kallas KM, Vaughan L, Haghighi P, Resnick D. Hibernoma of the left axilla; a case report and review of MR imaging. Skeletal Radiol 2003;32:290-4

11. Florio G, Cicia S, Del Papa M, Carni D. Neck hibernoma: Case report and literature review. G Chir 2000;21:339-41.

12. Wright C, Berry BH Jr, Patterson P, Griffiths H. Radiologic case study. Hibernoma. Orthopedics 2003;26:1194, 1268-70. 